Doug Geisler, Eva K. Grebel, and Dante Minniti, eds.

\title{
Systematic Properties of Young Clusters and Associations in Galaxies
}

\author{
Robert C. Kennicutt, Jr. \\ Steward Observatory, University of Arizona, Tucson, AZ 85721, USA
}

Fabio Bresolin

Institut für Physik und Astronomie, Ludwig-Maximilians-Universitat, Scheinerstrasse 1, D-81679, Munich, Germany

Andrew E. Dolphin

National Optical Astronomy Observatories, P.O. Box 26732, Tucson, $A Z$ 85726, USA

\begin{abstract}
We review what is known about the large-scale trends in the sizes, luminosities, and mass functions of young stellar associations and clusters in nearby galaxies. We also describe an ongoing survey of massive young cluster populations in nearby galaxies.
\end{abstract}

\section{Introduction}

Galaxies display an extraordinary diversity in their star formation properties along the Hubble sequence, with thousandfold ranges in their star formation rates (SFRs) per unit mass or area (Kennicutt 1998). To what extent does this variation reflect underlying trends in the clustering properties of the star formation? Information on the systematic variation of young cluster properties across the Hubble sequence are of obvious relevance to understanding the formation and evolution of star clusters themselves, but it is also critical to a wider range of problems including the structure of the ISM, the physical nature of the Hubble sequence, and the role of feedback from star formation and supernovae on the ISM and the evolution of galaxies.

This review will focus on what is known about the broad ensemble properties of young cluster and association properties (specific frequencies, size distributions, luminosity functions, etc) across the Hubble sequence. Such studies are handicapped by the effects of stellar crowding and limited spatial resolution for galaxies beyond the Local group (at the distance of the Virgo cluster the 30 Doradus core cluster subtends an angular extent of less than 0 "'1!), but this is balanced by the variety of observational tracers available. Apart from direct observations of resolved and unresolved stellar associations and clusters, the luminosity functions of the HII regions provide information on the mass distributions of the embedded star clusters, and HST observations can provide information on the ensemble properties of the clusters even when they are not resolved into stars. I will only briefly mention specific studies of local OB asso- 
ciation populations or the statistics of massive blue clusters in galaxies, as these are covered by the papers by Efremov and Larsen elsewhere in this volume.

\section{Diameters of OB Associations}

Young stellar associations and super-associations have characteristic dimensions (order $100 \mathrm{pc}$ ) that allow them to be identified to distances of order $10 \mathrm{Mpc}$ from the ground and $100 \mathrm{Mpc}$ with HST. In addition, the associations possess short dynamical lifetimes, ensuring that one is dealing with a uniformly young population, and thus simplifying the interpretation of the characteristic luminosities and sizes of these regions. A review of the groundbased work on nearby galaxies is given in Hodge (1988). These studies provided the first quantitative statistics on the size distributions and spatial distributions of young clusters, and quantitative evidence for a systematic change in young cluster properties with galaxy type (Wray \& de Vaucouleurs 1980, Hodge 1988 and references therein).

The sizes of the largest associations are of particular interest for star formation theory, because they provide constraints on the characteristic length scales for the ISM instabilities that lead to cloud and cluster formation. The most comprehensive study of the sizes of the largest associations has been carried out by Elmegreen et al. $(1994,1996)$. These authors used blue images from the Sandage \& Bedke (1988) atlas to identify the largest associations in 267 spiral and irregular galaxies, and to obtain visual diameter estimates. They found that among galaxies of the same type the diameter of the largest association increases with galaxy luminosity, following the trend expected if disk gravitational stabilities determine the characteristic length scale. However once this effect is removed the characteristic sizes do not appear to vary systematically with either galaxy type or spiral arm class. This is consistent with the gravitational stability picture, and suggests that the length scale for star forming complexes is insensitive to global properties of the parent galaxies, at least at the present epoch. It is notable that the diameters of the largest HII regions follow different trends, but this can be understood because the diameters of HII regions are more heavily influenced by the ionizing luminosities of the associations than by the sizes of the associations themselves.

When a stellar association is near enough to be well resolved one can use stellar statistics to determine its size in a more objective manner. The growing archive of HST observations has made it possible to extend this approach to galaxies with distances of $10 \mathrm{Mpc}$ and larger. Most recent applications to date have used variants of the automated techniques introduced by Battinelli (1991) and Wilson (1991), in which pairs and groups of stars within a specified search radius are identified automatically and grouped into clusters and associations ("friends-of-friends" algorithm). Recent applications of the technique include Wilson \& Bakker (1996), Bresolin, Kennicutt, \& Stetson (1996), Bresolin et al. (1998), and Williams \& Hodge (2001). Figure 1 shows a comparison of the size distributions derived for 7 nearby spiral galaxies observed with HST (Bresolin et al. 1998). It illustrates a common result of most of these studies, namely a rough consistency in the size distributions independent of parent galaxy type. This is reminiscent of the conclusions of Elmegreen et al. (1996) based on the statisitics of the largest associations in a larger sample of galaxies. One caveat to bear 


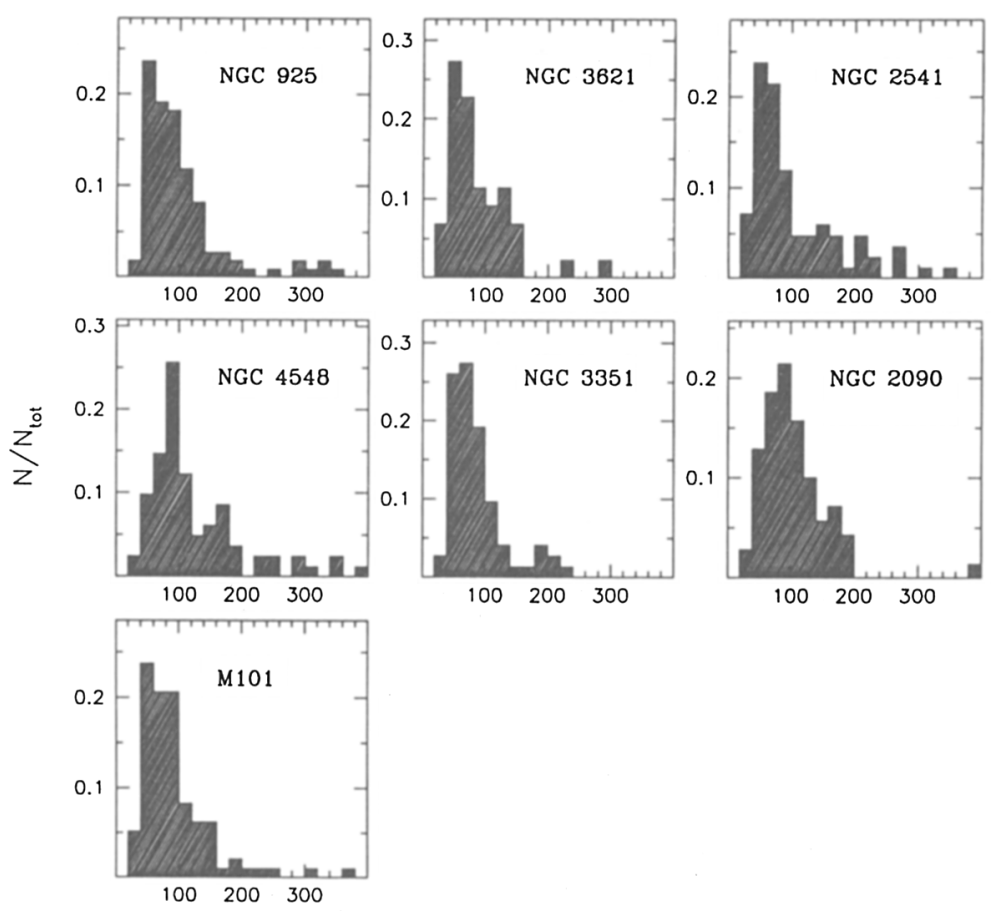

Figure 1. Comparison of $\mathrm{OB}$ association size distributions in 7 nearby galaxies, from Bresolin et al. (1998).

in mind with these studies is that the derived size distribution is sensitive to the search radius used, so subtle variations in the physical clustering spectrum might be partly masked in an automated search. However the current results clearly rule out gross changes in the size distributions. As will be seen below the behavior of the association luminosity functions is quite different.

\section{Luminosity Functions of OB/HII Associations}

Measuring accurate association luminosity functions (LFs) at visible wavelengths is difficult, because of the low contrast of the associations over the background disk, and because it can be difficult to separate effects of mass and age on the observed luminosities. Consequently the most reliable data on the luminosities of $\mathrm{OB}$ associations and young clusters come from measurements of the HII region luminosity function (LF). OB/HII regions are uniformly young objects $(\tau<5$ $\mathrm{Myr}$ ) and luminous HII regions require the composite ionization of multiple OB stars, so the shape of the nebular LF roughly traces the mass distribution of the stellar associations (Kennicutt 1988). The detailed shape of the LF can be influenced by age effects, deviations from strict ionization bounding, and 


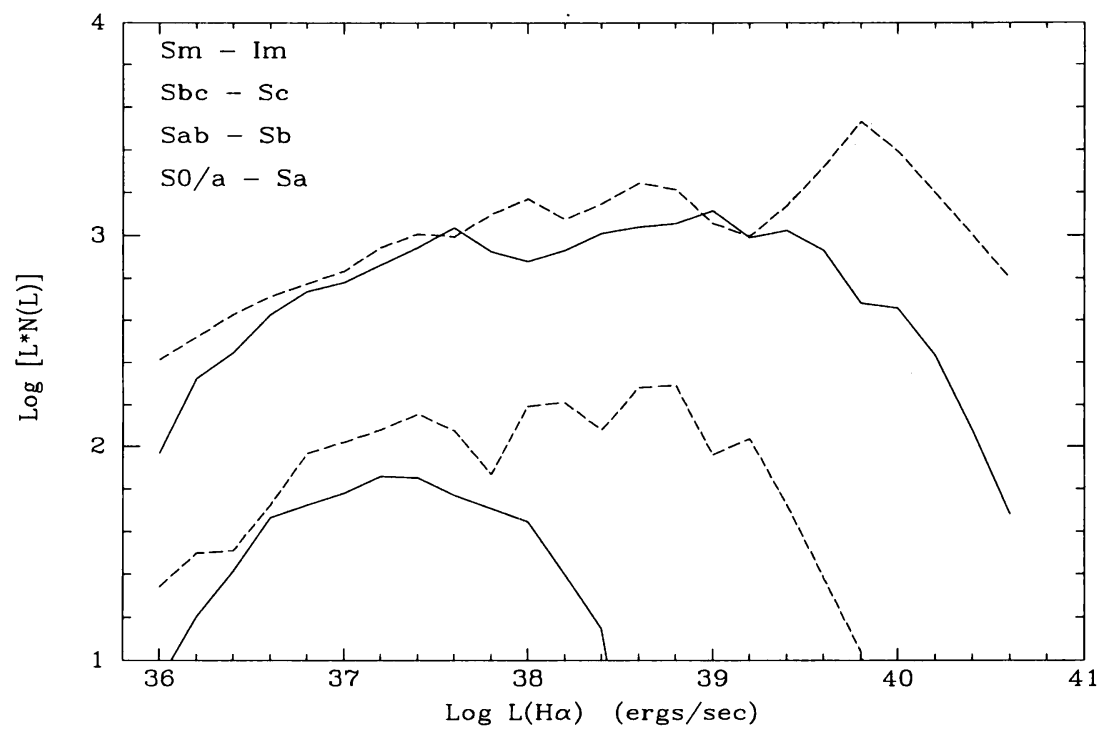

Figure 2. Composite HII regions LFs as a function of RSA galaxy type.

small number effects in the most massive stars (Oey \& Clarke 1998), so for this discussion we will concentrate on gross trends in the LFs with galaxy type.

Studies of the HII region $(\mathrm{H} \alpha)$ LFs of nearby galaxies have been published by numerous authors, with the most extensive studies including Kennicutt, Edgar, \& Hodge (1989), Caldwell et al. (1991), Banfi et al. (1993), Rozas, Beckman, \& Knapan (1996), Gonzalez-Delgado \& Perez (1997), Feinstein (1997), and Youngblood \& Hunter (1999). For bright objects $\left(L(H \alpha)>10^{37} \mathrm{ergs} \mathrm{s}^{-1}\right)$ the LF usually is well approximated by a power law with slope $d N / d L \sim-2 \pm 0.5$. In some objects there is evidence for a break in the function but this may be due to the combined effects of an upper cutoff in the LF, age variation, and/or crowding (Kennicutt et al. 1989, Oey \& Clarke 1998, Scoville et al. 2001).

In contrast to the $\mathrm{OB}$ association diameter distributions, the HII region LFs show a marked dependence on parent galaxy type. This is illustrated in Figure 2, which shows a compilation of LFs from the literature, binned by RSA galaxy type. The ordinate in the plot is the (differential) number of HII regions per logarithmic luminosity bin, normalized to constant $B$ luminosity, and is multiplied by luminosity so that equal area under the curve corresponds to equal total luminosity (or approximately equal cluster mass in the limit of multiple star ionization and a constant IMF vs type). As one progresses toward more actively star forming late-type galaxies one observes an increase in the number of starforming regions of all luminosities (when normalized to fixed galaxy luminosity or mass), plus a shift in the bulk of the star formation toward more luminous regions. Hence the increase in the SFR observed along the Hubble sequence is due to a combination of an increase in the frequency of star-forming events along with a shift in the mass spectra of at least the largest OB associations. 


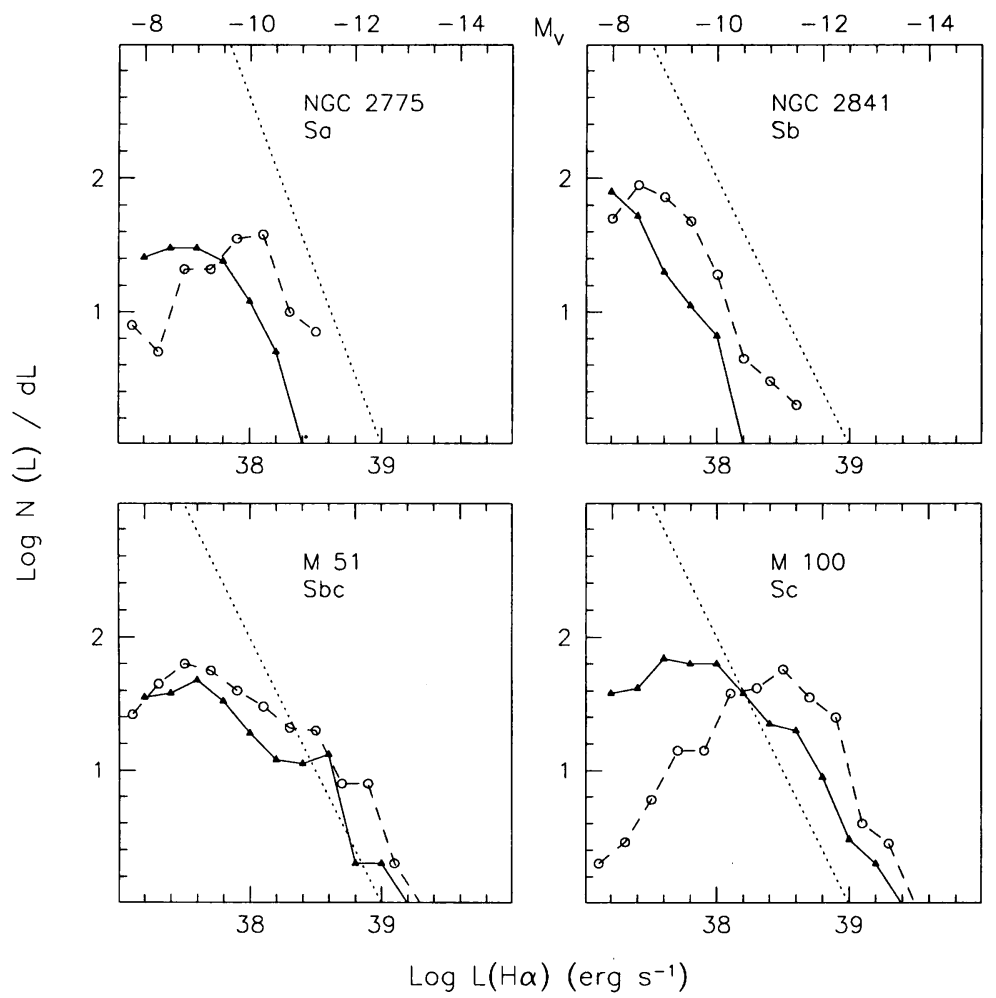

Figure 3. Continuum (dashed) and $\mathrm{H} \alpha$ (solid) LFs for the brightest knots and HII regions, respectively, in 4 galaxies. The dotted line shows a constant fiducial LF for reference. Adapted from Bresolin \& Kennicutt (1997).

The logarithmic scale in Figure 2 tends to mask the profound changes in the characteristic star formation environments across this sequence. In the earliesttype disks (S0/a-Sa) the typical OB star forms in a region like Orion, probably with several tens or hundreds of stars overall but with only a few other massive stars at most. Thus the effects of stellar winds and supernovae in such galaxies are likely to be imposed on the ISM in isolated cells, a few stars at a time. By contrast, in the Sc-Irr galaxies the most likely formation environment is a massive association on the scale of NGC 3603 in the Galaxy or 30 Dor in the LMC, with hundreds or thousands of other OB stars, and total cluster masses of order $10^{4} \mathrm{M}_{\odot}$ and above. The effects of stellar winds and SN in such galaxies are predominantly collective in nature; $\mathrm{SN}$ often will explode inside interstellar cavities formed by previous SN and stellar winds, and the character of the feedback on the ISM may be profoundly different. The effects of such collective feedback are seen directly in the X-ray structure and kinematics of these giant HII regions (e.g., Chu \& Kennicutt 1994, Martin 1998), but their large-scale effects on the ISM and galaxy evolution are only beginning to be understood. 
To what extent can the HII region LFs be trusted to trace the overall luminosity and mass functions of the OB associations? The trend seen in Figure 2 could be produced, for example, by a strong gradient in the upper IMF in the clusters along the Hubble sequence. A test would be to measure the visible continuum LFs for the same associations. The latter are much less sensitive to IMF variations than the $\mathrm{H} \alpha$ luminosities, which effectively measure the cluster fluxes below the Lyman limit. Such a test was carried out by Bresolin \& Kennicutt (1997), who used $U B V R$ and $\mathrm{H} \alpha$ photometry of 10 galaxies to measure continuum and nebular LFs on a self-consistent basis. They found that the continuum LFs generally follow the shape of the $\mathrm{H} \alpha \mathrm{LFs}$, and that the continuum LFs show the same strong changes along the Hubble sequence (see Figure 3). Comparisons of the $\mathrm{H} \alpha$ and red continuum luminosities of the brightest HII regions in a larger sample of galaxies shows the same trends. These results rule out IMF variations as the dominant source of the LF changes, though a secondary contribution to the changes cannot be ruled out. Apparently the LF changes reflect actual changes in the clustering properties of the associations themselves.

\section{Implications for the Formation of Globular Clusters}

Apart from comparing the shapes of the HII region and association LFs, we can apply the specific frequencies of the young objects to estimate the fraction of star forming regions that may produce a long-lived populous cluster. For luminous HII regions the ionizing luminosity scales directly with the cluster mass (at least for the massive stars), and conversions between $\mathrm{H} \alpha$ luminosity and cluster mass are found in Kennicutt (1988) and Leitherer et al. (1999). With such information and the LFs discussed earlier it follows that the massive cluster/associations form at a rate that is $2-3$ orders of magnitude higher than can be explained by the number of intermediate-age populous clusters. For example the Galaxy produces a cluster/association of at least $10^{4} \mathrm{M}_{\odot}$ every few million years (at a minimum), and if all of these objects survived, the disk of the Galaxy should contain at least 1000 such clusters; at most there are a few. A more rigorous calculation (Kennicutt \& Chu 1988) shows that this result holds generally among nearby galaxies, and the discrepancy cannot be attributed to luminosity evolution effects or other selection effects in the cluster samples.

In many ways this is a recapitulation of a decades-old result, namely that OB associations in the Galaxy dissolve on timescales of a few tens of Myr. However these observations imply further that most of the "blue globular clusters" or "super star clusters" being formed in giant HII regions- $95 \%$ or more- will not survive past ages of order 0.1-1 Gyr either. The dominant evolutionary process for young clusters in galaxies is cluster destruction! The precursors to the halo globular clusters in our own Galaxy and others must have been quite rare and extraordinary objects. Massive bound clusters still are being formed in the Magellanic Clouds and other galaxies, so we know that some local star formation regions harbor such objects. However it would be difficult to predict a priori which few percent of recently formed clusters and associations will produce long-lived globular clusters. 


\section{Properties of Massive Young Star Clusters}

Although it is tempting to associate the results described above with the differences in blue cluster populations discussed elsewhere at this symposium, it is important to emphasize that the results just presented apply mainly to OB/HII associations. To be sure many of these associations (e.g., 30 Doradus) appear to contain the progenitors of massive bound clusters (e.g., Kennicutt \& Chu 1988), but it is important to establish directly whether the trends described above apply to bound star clusters as well.

An impressive attack on this problem has been made by Larsen \& Richtler $(1999,2000)$, as reported in detail by Larsen elsewhere in this volume. Groundbased imaging of 21 nearby galaxies has been analyzed with automated stellar photometry software to identify and to catalog bright young clusters, and correlate the properties of the cluster populations with large-scale properties of the parent galaxies. In particular these authors observe an increase in the fraction of star formation contained in large clusters with galaxy type, which they attribute to an underlying correlation with the SFR.

Currently we are extending this approach by carrying out a similar analysis of a large sample of galaxies observed with HST, many of them as part of the various Cepheid variable campaigns. A detailed description of this program is given in Dolphin \& Kennicutt (2001) and we only summarize the highlights here. Even with HST populous clusters are marginally resolved beyond a distance of a few $\mathrm{Mpc}$, so we are restricting our initial analysis to galaxies with distances in the $10-20 \mathrm{Mpc}$ range, and with sufficient integration times (typically $40,000 \mathrm{~s}$ and $20,000 \mathrm{~s}$ in $V$ and $I$ respectively) to be able to carry out a robust star/cluster separation. The photometry is being carried out using the HSTphot package of Dolphin (2000), with star cluster discrimination based mainly on image sharpness and magnitude. A key part of the analysis is a comprehensive set of artificial cluster simulations, in which synthetic clusters are added to images and extracted to robustly test the efficiency of cluster detection and any biases that might arise as functions of cluster age, luminosity, size, or local crowding.

Table 1 summarizes our preliminary results for three galaxies. A new Cepheid distance to each has been calculated using the HSTphot photometry; all of the recovered distances are compatible with results of earlier studies. The cluster candidates were then corrected for photometric errors using the results of the artificial cluster tests, providing true luminosity functions and CMDs.

Of the three galaxies, NGC 3627 is the only to contain large numbers of very bright $\left(M_{V}<-8\right)$ cluster candidates. For those that fall in the planetary camera chip, we measure typical core radii of $\sim 1 \mathrm{pc}$ and colors of $(V-I)<0.5$ - parameters comparable to young LMC clusters. In all three galaxies, the blue clusters trace the spiral arms (as well as the bar of NGC 4639) while the red clusters are distributed more evenly. NGC 4527 is the only of the four with significant halo in the WFPC2 field of view, and for that galaxy we find many likely halo globular clusters. For the set of three galaxies, we find that the cluster luminosity functions are consistent in the three objects, although the luminosity of any one cluster is a strong combination of its initial mass and age and it is difficult to disentangle these two factors. 
Table 1. Preliminary Results

\begin{tabular}{lccc}
\hline Quantity & NGC 3627 & NGC 4527 & NGC 4639 \\
\hline Type & Sb & Sbc & SBb \\
$V$ time & $35000 \mathrm{~s}$ & $55000 \mathrm{~s}$ & $60000 \mathrm{~s}$ \\
$I$ time & $15000 \mathrm{~s}$ & $25000 \mathrm{~s}$ & $12500 \mathrm{~s}$ \\
$\mu_{0}(\mathrm{mag})$ & $30.35 \pm 0.18$ & $30.81 \pm 0.14$ & $31.81 \pm 0.10$ \\
$\mu_{0}^{1}(\mathrm{mag})$ & $30.28 \pm 0.15^{1}$ & $30.74 \pm 0.12^{2}$ & $32.03 \pm 0.22^{3}$ \\
$\mu_{0}^{2}(\mathrm{mag})$ & $30.06 \pm 0.17^{4}$ & $30.67 \pm 0.15^{5}$ & $31.80 \pm 0.09^{4}$ \\
$A_{V}(\mathrm{mag})$ & 0.11 & 0.40 & 0.09 \\
Cluster LF, $M_{V}<-10$ & $-2.5 \pm 0.8$ & $\mathrm{n} / \mathrm{a}$ & $\mathrm{n} / \mathrm{a}$ \\
Cluster LF,$-10<M_{V}<-8$ & $-1.0 \pm 0.3$ & $-1.0 \pm 0.2$ & $-1.0 \pm 0.2$ \\
Ctellar LF, $-10<M_{V}<-8$ & $-3.4 \pm 0.5$ & $-2.2 \pm 0.6$ & $-2.2 \pm 0.3$ \\
\hline \hline
\end{tabular}

${ }^{1} \mathrm{SNe}$ Ia project distance moduli: NGC 3627 (Saha et al. 1999), NGC 4527 (Saha et al. 2001), and NGC 4639 (Saha et al. 1997).

${ }^{2} \mathrm{H}_{0}$ Key Project distance moduli: NGC 3627 and NGC 4639 (Gibson et al. 2000) and NGC 4527 (Calculated from the Gibson \& Stetson 2001 photometry, using the Madore \& Freedman 1991 P-L relations)

\section{Concluding Remarks}

Taken together these results show that the characteristic masses and luminosities of young clusters and associations can change dramatically in different galactic environments, while the characteristic sizes of the star-forming regions appear to be relatively insensitive to these changes in environment. These results are consistent with the longstanding observations of different cluster populations in the Galaxy and the Magellanic Clouds; the latter reflect a more general change in clustering properties along the Hubble sequence. However the data are too sparse at this stage to allow us to isolate unambiguously the physical mechanisms that are responsible for the changes. Solving this problem will be critical to understanding the cluster populations in galaxies in the larger context, and to quantifying the extent to which the star clustering may regulate the feedback of stars on the ISM and galaxy evolution as a whole.

Acknowledgments. The research presented here was supported by the National Science Foundation through grant AST-9900789, by NASA/STScI grants GO-02227.06-87A and HST-AR-09196.01-A, and NASA grant NAG5-8426.

\section{References}

Banfi, M., Rampazzo, R., Chincarini, G., \& Henry, R.B.C. 1993, A\&A, 280, 373 Battinelli, P. 1991, A\&A, 244, 69

Bresolin, F., Kennicutt, R.C., \& Stetson, P.B. 1996, AJ, 112, 1009

Bresolin, F., \& Kennicutt, R.C. 1997, AJ, 113, 975

Bresolin, F. et al. 1998, AJ, 116, 119 
Caldwell, N., Kennicutt, R., Phillips, A.C., \& Schommer, R.A. 1991, ApJ, 370, 526

Chu, Y.-H., \& Kennicutt, R.C. 1994, ApJ, 425, 720

Dolphin, A.E. 2000, PASP, 112, 1383

Dolphin, A.E., \& Kennicutt, R.C. 2001, in ASP Conf Ser, Modes of Star Formation and the Origin of Field Populations, ed. E.K. Grebel \& W. Brandner (San Francisco: ASP), in press

Elmegreen, D.M., Elmegreen, B.G., Lang, C., \& Stephens, C. 1994, ApJ, 425, 57

Elmegreen, B.G., Elmegreen, D.M., Salzer, J.J., \& Moran, H. 1998, ApJ, 467, 579

Feinstein, C. 1997, ApJS, 112, 29

Gibson B.K. et al. 2000, ApJ, 529, 723

Gibson, B.K. \& Stetson, P.B. 2001, ApJ, 547, 103

Gonzalez-Delgado, R.M., \& Perez, E. 1997, ApJS, 108, 199

Hameed, S., \& Devereux, N. 1999, AJ, 118, 730

Hodge, P.W. 1988, PASP, 100, 568

Kennicutt, R.C. 1988, ApJ, 334, 144

Kennicutt, R.C., \& Chu, Y.-H. 1988, AJ, 95, 720

Kennicutt, R.C. 1998, ARAA, 36, 189

Kennicutt, R.C., Edgar, B.K., \& Hodge, P.W. 1989, ApJ, 337, 761

Larsen, S.S., \& Richtler, T. 1999, A\&A, 345, 59

Larsen, S.S., \& Richtler, T. 2000, A\&A, 354, 836

Leitherer, C. et al. 1999, ApJS, 123, 3

Madore, B.F., \& Freedman, W.L. 1991, PASP, 103, 933

Martin, C.L. 1998, ApJ, 506, 222

Oey, M.S., \& Clarke, C.J. 1998, AJ, 115, 1543

Rozas, M., Beckman, J.E., \& Knapen, J.H. 1996, A\&A, 307, 735

Saha, A. et al. 1997, ApJ, 486, 1

Saha, A. et al. 1999, ApJ, 522, 802

Saha, A. et al. 2001, ApJ, 551, 973

Sandage, A., \& Bedke, J. 1988, Atlas of Galaxies (Washington:GPO)

Scoville, N. et al. 2001, ApJ, submitted

Williams, B., \& Hodge, P.W. 2001, ApJ, submitted

Wilson, C.D. 1991, AJ, 101, 1663

Wilson, C.D., \& Bakker, K.J. 1996, AJ, 112, 1588

Wray, J.D., \& de Vaucouleurs, G. 1980, AJ, 85, 1

Youngblood, A.J., \& Hunter, D.A. 1999, ApJ, 519, 55 


\section{Discussion}

P. Barmby: If your interpretation of the GCLF differences between the metalrich and metal-poor populations is correct, this would imply that the cluster mass distributions are nearly identical. Isn't this somewhat surprising given the very different formation and dynamical environment for the two sub-populations?

R. Kennicutt: Yes, it probably would be surprising. The GCLF just doesn't give enough leverage to figure out what combination of age/ mass/ IMF differences might really exist between metal-rich and poor clusters, so we just picked one (age) as the simplest possibility.

J. Holtzman: How are we to interpret the trends in the HII region luminosity functions with Hubble type when the Hubble classification is based in part on the properties of the star forming regions?

R. Kennicutt: This is an excellent point. One sees the strongest trends in star formation properties when they are correlated with RSA type; this is not surprising because Sandage gives heavy weight to disk resolution when he assigns a spiral type. However the trends are still present, albeit with more scatter, when RC3 types are used (which are more heavily based on bulge/disk ratio). In the specific case of the HII region luminosity functions, the increase in total number of HII regions with type may be partly built into the classification. However the change in the shape of the LF with type demonstrates a fundamental correlation between galaxy type (or SFR) and clustering that holds regardless of how we classify galaxies. An excellent discussion is found in Hameed \& Devereux (1999).

J. Melnick: Did you check if extinction and metallicity effects could explain the differences in HII region luminosity functions along the Hubble sequence?

R. Kennicutt: Yes, we have extinction and/or reddening data for many of these objects, and while there is a considerable variation in extinction from object to object and a very weak trend with galaxy type, the trends are far too small to explain the LF changes. As for metallicity, there is a general decrease in abundance along the Hubble sequence that may contribute directly or indirectly to the LF changes we see. However we have tested more directly for abundance effects by looking for a radial variation in the LFs within individual galaxies with strong abundance gradients. No significant radial dependence in the LF is seen, which suggests that metallicity effects are unimportant, relative to the change in the clustering mass spectrum itself.

W. Harris: The point that very few of the young objects in HII regions and disk clusters will survive over the long term $(<1 \%)$ is nicely argued. To the extent that the numbers of old clusters in E galaxies represent the "super star clusters" that did survive, recent papers by McLaughlin, Blakeslee, and others show that the globular clusters represent about 0.0025 of the stellar mass in these galaxies, quite consistently. That is, only $1 / 4$ of one percent of the formed stars are left 
in long-lasting objects.

R. Kennicutt: I am gratified to hear that my rough estimate of a $0.1-1 \%$ survival fraction is consistent with a rigorous analysis! I do believe that the numbers are directly comparable, because recent work on star formation in the Galaxy seems to suggest that the bulk of stars, even those that end up in the field, form in clusters of some kind.

J. Frogel: O'Connell \& De Gris say M82 has some $130 \sim 500$ Myr-old clusters like NGC 1866. You claimed these should have evaporated. Please explain.

R. Kennicutt: The results I quoted refer to the relatively quiescent star formation that takes place in the extended disks of spiral and irregular galaxies. The physical conditions in the dense inner molecular disks of infrared-luminous starburst galaxies and mergers are quite different (see the review by Kennicutt 1998), and it is quite possible (I would say even likely) that those conditions are more favorable to the formation of globular clusters. Having said that, however, I do not think we should be too hasty in assuming that the M82 clusters will survive indefinitely. The age estimates are approximate and stellar mass loss alone may cause many of them to dissolve before they reach an age of 1 Gyr.

J. Maiz-Apellaniz: The bad news may not be so bad after all. Of all the nearby massive young clusters, the R136 cluster in 30 Doradus is actually the most compact of all. So resolving massive young clusters within a few tens of $\mathrm{Mpc}$ may be possible after all.

R. Kennicutt: I fully agree, the work by Larsen \& Richtler and our own preliminary work shows that it is possible to identify compact clusters, mainly by using the structural signatures of the more extended stellar distributions. My main point was to caution that you cannot always uniquely associate these young "super clusters" with bona-fide globular clusters based on structural criteria alone. As the discussion above emphasizes, most of the young objects cannot form long-lived globular clusters.

H. Zinnecker: You seem to be convinced of the universality of the (Salpeter) IMF in extragalactic star forming regions. Please remind us on which observations this conclusion is based. After all, some people at this conference have claimed big IMF variations.

R. Kennicutt: I would not go so far as to say that the observations rule out any IMF variations - what I would say is that the spectroscopic properties and spectral energy distributions of the young clusters/associations rule out the kinds of enormous variations in the IMF that would be required to explain the changes in the luminosity functions of the HII regions and associations in normal galaxies. Probably the strongest hints for large IMF variations are for the dense massive clusters in starburst galaxies like M82, as discussed in detail elsewhere at this conference. However I know of no strong evidence for such variations in the star clusters of the Milky Way or other normal galaxies. 